\title{
Study of a scalable large-area radio-frequency helicon plasma source
}

\author{
T. Windisch ${ }^{1}$, K. Rahbarnia ${ }^{1,2}$, O. Grulke ${ }^{1,2}$, T. Klinger ${ }^{1,2}$ \\ ${ }^{1}$ Max-Planck-Institute for Plasma Physics, EURATOM Association, D-17491 \\ Greifswald, Germany \\ ${ }^{2}$ Ernst-Moritz-Arndt University, D-17491 Greifswald, Germany \\ E-mail: thomas.windisch@ipp.mpg.de
}

\begin{abstract}
A large-area planar spiral antenna has been installed on the linear laboratory device VINETA. For low radio-frequency $(R F)$ powers the induction coil is operated in the inductive discharge mode with typical plasma densities of $n \approx 1 \cdot 10^{16} \mathrm{~m}^{-3}$ and electron temperatures of $T_{e} \approx 5 \mathrm{eV}$. At higher $R F$ powers a transition to the helicon wave sustained discharge mode is observed, in which the density increases by a factor of 200 and $T_{e}$ drops by a factor of 2 . Detailed measurements of the helicon wavefield show that a helicon wave with azimuthal modenumber $m=0$ is launched. Due to the larger wave excitation region of the $m=0$ discharges the width of the plasma density profile in the helicon mode increases by a factor of 1.5 compared the standard VINETA helicon operation with a helical $m=+1$ antenna.
\end{abstract}

PACS numbers: $52.50 .-\mathrm{b}, 52.40 . \mathrm{Fd}$

Keywords: electro-magnetic fields, radio-frequency plasma sources, inductively coupled plasma, Helicon waves, 


\section{Introduction}

Radio-frequency $(R F)$ discharges are commonly used for plasma production in industrial applications $[1,2]$ and laboratory devices $[3,4,5]$ at low $R F$ power $\left(P_{R F}<5 \mathrm{~kW}\right)$. In high $R F$ power operation $\left(P_{R F} \approx 150 \mathrm{~kW}\right)$ they have become attractive, e.g. as negative ion sources for the neutral beam injection heating of magnetic fusion devices [6] or for space craft propulsion using plasma thrusters [7]. Depending on the antenna geometry, boundary conditions and operational constraints, $R F$ sources can be operated in either capacitive, inductive or helicon wave sustained discharge modes [8], which gives access to a broad parameter range for plasma density, temperature and neutral gas pressure. Especially helicon wave sustained discharges attracted much interest since their discovery [9, 10] by Boswell in the mid 1980's because plasma densities up to $10^{19} \mathrm{~m}^{-3}$ can easily be achieved with $2-3 \mathrm{~kW}$ of $R F$ power. In cylindrical geometry, helicon waves develop an eigenmode structure. In order to excite helicon modes with azimuthal modenumber $m=0$ or $m=1$ specific antenna geometries have been developed [11, 12]. The helicon wave excitation region is controlled by the plasma geometry, i.e, the radial width of the plasma density profile. The latter is given by the specific antenna geometry. Conventional helicon sources mostly use a right-handed $m=+1$ helical antenna with a fixed radius. As a result the radial shape of the plasma density profile cannot be changed during operation without facing technical difficulties. In order to overcome these obstacles a planar spiral antenna is used for plasma production. Given by it's geometry, the spiral antenna preferably excites a $m=0$ helicon mode for high $R F$ powers, while at lower power the discharge is mainly inductive. Detailed investigations of plasma production using a $m=0$ helicon antenna have been carried out by Shinohara and co-workers, e.g. about the spatial helicon wave structure and dispersion [3] and the influence of the magnetic field configuration and antenna field pattern on the radial density profile [13]. On the VINETA device, which normally uses a conventional $m=+1$ antenna (diameter of $10 \mathrm{~cm}$ ) [14], such a planar induction antenna with a diameter of $20 \mathrm{~cm}$ has been recently installed in order two address two key issues. First, to produce homogeneous density profiles at reduced Coulomb collisional using the inductive discharge mode. This issue is important for the study of various dynamical plasma processes, which are strongly affected by collisional damping. Secondly, to increase the plasma radius compared to the VINETA standard configuration in the helicon discharge mode. Power connections at different radial positions of the antenna allow one to control the helicon wave absorption location.

This paper is organized as follows: In Sec. 2 the experimental and diagnostic setup are described. Two-dimensional plasma parameter profiles in the inductive mode are discussed in Sec. 3. The transition from the inductive to the helicon wave sustained mode is described in Sec. 4, as well as the characteristics of the helicon wave. The results are summarized in Sec.5.

\section{Experimental setup and discharge characteristics}

The experiments were conducted in the magnetized linear plasma device VINETA [4].A schematic drawing is shown in Fig. 1. The device consists of four identical modules and has a total length of $4.5 \mathrm{~m}$. The cylindrical stainless steel vacuum vessel has a diameter of $0.4 \mathrm{~m}$ and is immersed in a set of 36 magnetic field coils, providing a homogeneous magnetic field $B_{0}$ up to $100 \mathrm{mT}$. In the standard 


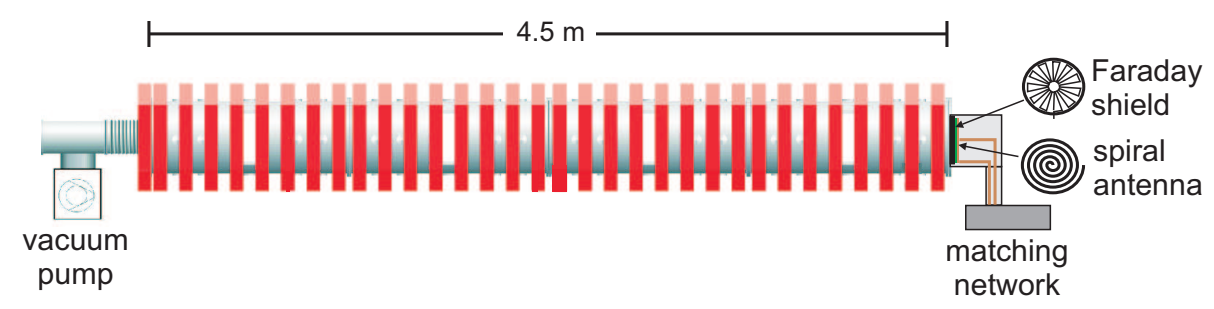

Figure 1. Schematic diagram of the VINETA device using the spiral antenna as plasma source.

configuration, the plasma is produced using a helical $m=+1$ antenna [15] at the one end of the device. The antenna is placed around a cylindrical glass vacuum extension $(d=0.1 \mathrm{~m}, l=0.5 \mathrm{~m})$ and is connected to a $R F$-source $\left(f_{R F}=13.56 \mathrm{MHz}\right.$, $P \leq 4 \mathrm{~kW})$ via a L-matching circuit [16]. The measurements presented here are made with Argon as working gas. Time-averaged plasma parameters are measured with Langmuir probes compensated against $R F$ potential fluctuations [17]. Plasma density, electron temperature and plasma potential are extracted from the probe characteristics using a kinetic probe theory that takes into account the ambient magnetic field $B_{0}[18]$. For the high density (helicon) discharges, a $160 \mathrm{GHz}$-interferometer is used to crosscalibrate the plasma density as obtained from the probe characteristics. Magnetic fluctuations of the $R F$ field are measured using magnetic induction resp. $\dot{B}$-probes [19]. Our $\dot{B}$-probe consists of a loop antenna with four windings of $4 \mathrm{~mm}$ diameter, that are insulated from the plasma by a thin ceramic layer. For the measurements reported here the surface normal of the loop antenna is aligned parallel to the symmetry axis of the cylindrical device. $\dot{B}$-probes are known to react sensitively on $R F$-potential fluctuations. In order to reduce this capacitive pickup [20] they are manufactured using center-tapped transformers. This has been found to be the proper rejection method for VINETA plasma conditions [19]. $\dot{B}$ - and Langmuir probes are mounted on probe positioning systems, that move the probe computer controlled in the radialaxial plane.

Typical plasma parameters of the routine helicon discharges are compiled in Table 1. As typical for helicon discharges, a rather dense plasma with plasma densities up to $2 \cdot 10^{19} \mathrm{~m}^{-3}$ at low electron temperatures $T_{e} \leq 3 \mathrm{eV}$ is produced (the ions are cold with $T_{i} \approx 0.2 \mathrm{eV}$ ). The high plasma densities lead to a fairly high (normalized) plasma pressure $\beta \approx 1 \%$ and, together with low electron temperatures, to high Coulomb collision frequencies which dominate over collisions with neutrals in the

\begin{tabular}{llll} 
parameter & $\begin{array}{l}\text { standard helicon } \\
(m=+1)\end{array}$ & spiral ICP & unit \\
\hline$R F$ power & 1 & 0.6 & $\mathrm{~kW}$ \\
Argon neutral gas pressure $p_{0}$ & 0.4 & 0.4 & $\mathrm{~Pa}$ \\
plasma density $n$ & 5 & 0.02 & $10^{18} \mathrm{~m}^{-3}$ \\
electron temperature $T_{e}$ & 2.5 & 5 & $\mathrm{eV}$ \\
Coulomb collision frequency $\nu_{e i}$ & 37 & 0.04 & $10^{6} \mathrm{~s}^{-1}$ \\
electron-neutral collision frequency $\nu_{e n}$ & 3 & 10 & $10^{6} \mathrm{~s}^{-1}$
\end{tabular}

Table 1. VINETA plasma parameters. 


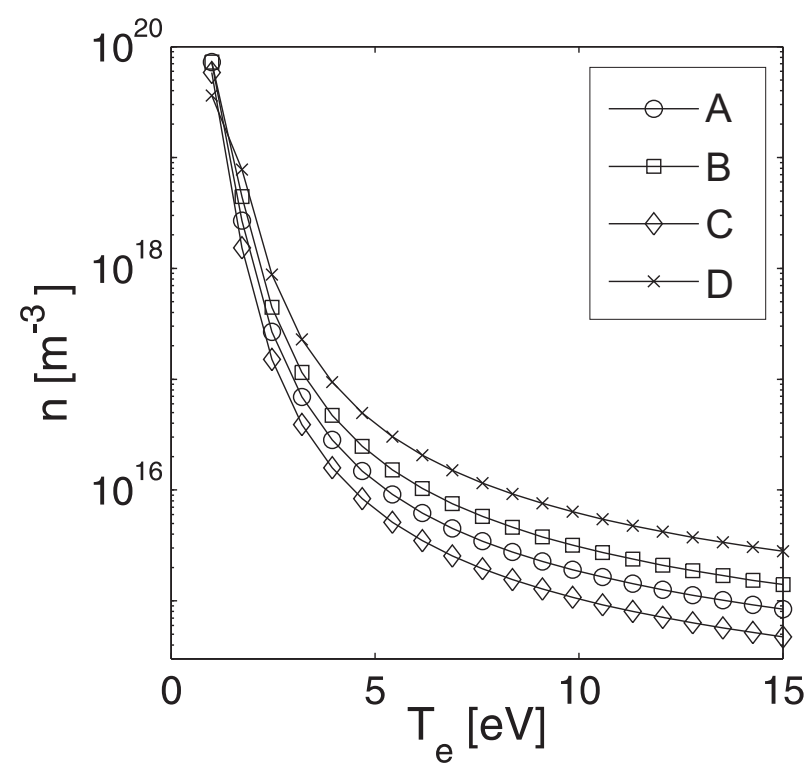

Figure 2. Result of the power balance calculation for different Argon neutral gas pressures $p 0$, input powers $P_{R F}$ and plasma radii $r_{p}$ : (A) $p_{0}=0.4 \mathrm{~Pa}, P_{R F}=600 \mathrm{~W}, r_{p}=60 \mathrm{~mm}(\mathrm{~B}) p_{0}=0.4 \mathrm{~Pa}, P_{R F}=1000 \mathrm{~W}$, $r_{p}=60 \mathrm{~mm}(\mathrm{C}) p_{0}=0.4 \mathrm{~Pa}, P_{R F}=600 \mathrm{~W}, r_{p}=80 \mathrm{~mm},(\mathrm{D}) p_{0}=0.2 \mathrm{~Pa}$, $P_{R F}=1000 \mathrm{~W}, r_{p}=60 \mathrm{~mm}$.

plasma center. The plasma density profile of the helicon plasma has a typical $1 / e$ folding length of $r_{p} \approx 40 \mathrm{~mm}$ (gradient length $L_{\perp}=n / \nabla n \approx 1 \mathrm{~cm}$ ). For lower $R F$ input powers the helical antenna can be operated either in capacitive or inductive mode [14], but these discharge modes have certain drawbacks, i.e., the capacitive mode is characterized by a hollow density profile and the inductive mode is only established at higher neutral gas pressures, which increases the neutral collision rate. To produce plasmas with homogeneous density profile at low collisionalities, the helical $m=+1$ antenna was replaced by a large-area spiral antenna [21]. This planar induction coil has five turns with an outer radius of $r_{a}=10 \mathrm{~cm}$ (Fig. 1) and is separated from the plasma by a quartz window. Similar to the helical antenna the planar spiral antenna can be operated in different modes [4]. If the absorbed $R F$-power is small, capacitive power coupling ( $E$-mode) dominates, while for higher $R F$-powers inductive coupling ( $H$-mode) becomes accessible $[22,23]$. The $E-H$-transition occurs if the skin depth $\delta \sim 1 / \sqrt{n}$ is close to the lateral dimension of the plasma. Since the inductance of the antenna is not perfect, capacitive coupling can still contribute, also at moderate $R F$-power. In order to reduce the capacitive coupling, which may lead to bi-Maxwellian electron energy distribution functions (EEDF) due to the trapping of low-energy electrons [24], a spoked grounded Faraday shield was placed between the quartz window and the induction coil [25]. It attenuates the $R F$ electric field caused by the large antenna potential while the magnetic fields can freely penetrate into the plasma. The typical plasma parameters in the spiral ICP discharges are shown for comparison in Table 1 as well. The plasma density $\left(n \approx 10^{16} \mathrm{~m}^{-3}\right)$ is significantly lower if compared to the routine helicon operation, while the electron temperature 
is a factor of two higher with $T_{e} \approx 5 \mathrm{eV}$. As a consequence, the Coulomb collision frequency $\nu_{e i}$ decreases by a factor of 100 and the electron-neutral collision frequency $\nu_{e n}$ increases by a factor of 3 .

The drop in the plasma density can be understood considering balance of particle and energy fluxes. Taking the main energy loss channels into account (elastic and inelastic collisions between electrons and ions, parallel losses and ionization) an expression for the plasma density as a function of the electron temperature can be derived from the rate equations [26]. The result is shown in Fig. 2 for different values for $R F$-power $R_{r f}$, Argon neutral gas pressure $p_{0}$, and plasma radius $r_{p}$. As a universal feature, the plasma density decreases for higher electron temperatures. This is caused by the power transport that is determined by the convective transport losses $3 / 2 T_{e} \Gamma_{n}=3 / 2 T_{e} V\left\langle\sigma_{i o n} v\right\rangle n n_{0}$, in which the ionization rate $\left\langle\sigma_{i o n} v\right\rangle$ depends on $T_{e}$ and $n$. Here, $V$ denotes the plasma volume, $n_{0}$ the neutral density and $\Gamma_{n}$ the particle flux. For a given electron temperature and a specific working gas, the plasma density can be increased by lowering the neutral gas pressure. Taking the electron temperatures and $R F$ input power from Table 1 as a reference value, one estimates from the power balance a plasma density of $n \approx 10^{18} \mathrm{~m}^{-3}$ for the standard helicon mode and $n \approx 10^{16} \mathrm{~m}^{-3}$ for the spiral ICP discharges, which agree well with measurements discussed in the next section.

The measured component of the magnetic field $B_{z}(r, z)$ in the radial-axial plane
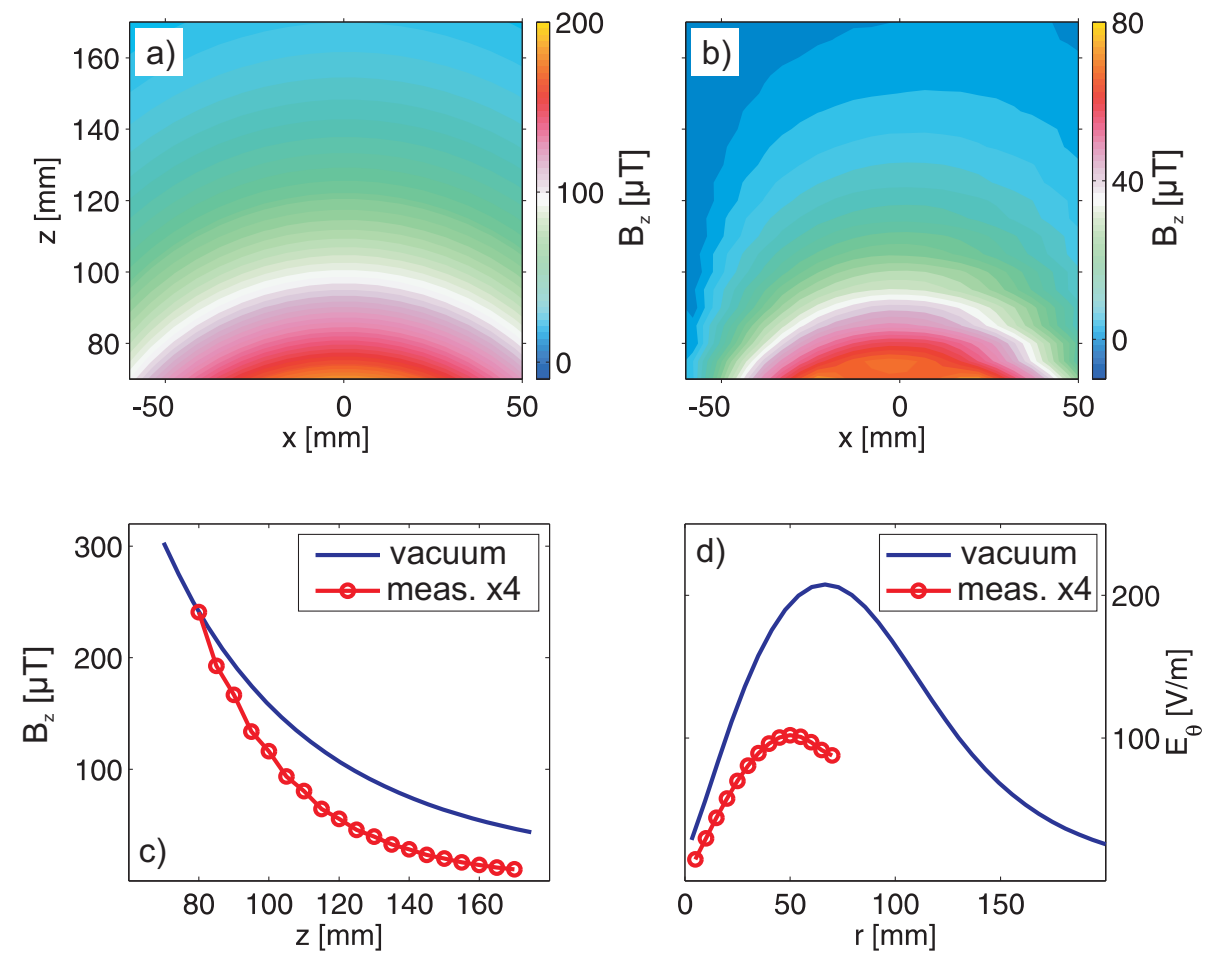

Figure 3. Calculated (a) and measured (b) magnetic field $B_{z}(r, z)$ in the radial-axial plane (c) and on the central axis $x=0$ (c). The antenna is located at $z=0$. (d) The resulting induced azimuthal eletric field $E_{\theta}(r, z)$ at $z=80 \mathrm{~mm}$. 
$(r, z)$ as created by the spiral antenna is shown in Fig. 3(b). For comparison the calculated vacuum magnetic field of the antenna is shown in Fig. 3(a) for an antenna current of $I=15 \mathrm{~A}$. The position $z=0$ corresponds to the antenna position. Since the electron plasma frequency $\omega_{p, e} \sim \sqrt{n}$ is larger than the $R F$-frequency there is a cutoff layer in the plasma. A much stronger damping of $B_{z}$ in the case with plasma is seen in Fig. 3c that shows the amplitude of $B_{z}(r=0, z)$ on the central axis. The measured exponential decay length decreases from $50 \mathrm{~mm}$ for the vacuum case to $27 \mathrm{~mm}$ with plasma. The latter value is close to the collisionless skin depth $\delta_{p}=c / \omega_{p e}=37 \mathrm{~mm}$ $\left(\nu_{e n} \ll \omega\right)$, estimated for the typical plasma density $n \approx 2 \cdot 10^{16} \mathrm{~m}^{-3}$ (cf. $4(\mathrm{a})$ ) of the spiral ICP discharges. The $R F$-power absorption is determined by the induced azimuthal electric field component $E_{\theta}(r, z)$, via

$$
\left\langle d P_{R F} / d V\right\rangle=\sigma\left\langle E_{\theta}^{2}\right\rangle
$$

where $\sigma$ is the plasma conductivity. Using Faraday's law and assuming cylindrical symmetry the azimuthal electric field $E_{\theta}(r, z)$ is given by

$$
E_{\theta}(r, z)=\frac{\omega}{r} \int_{0}^{r} r^{\prime} B_{z}\left(r^{\prime}, z\right) d r^{\prime}
$$
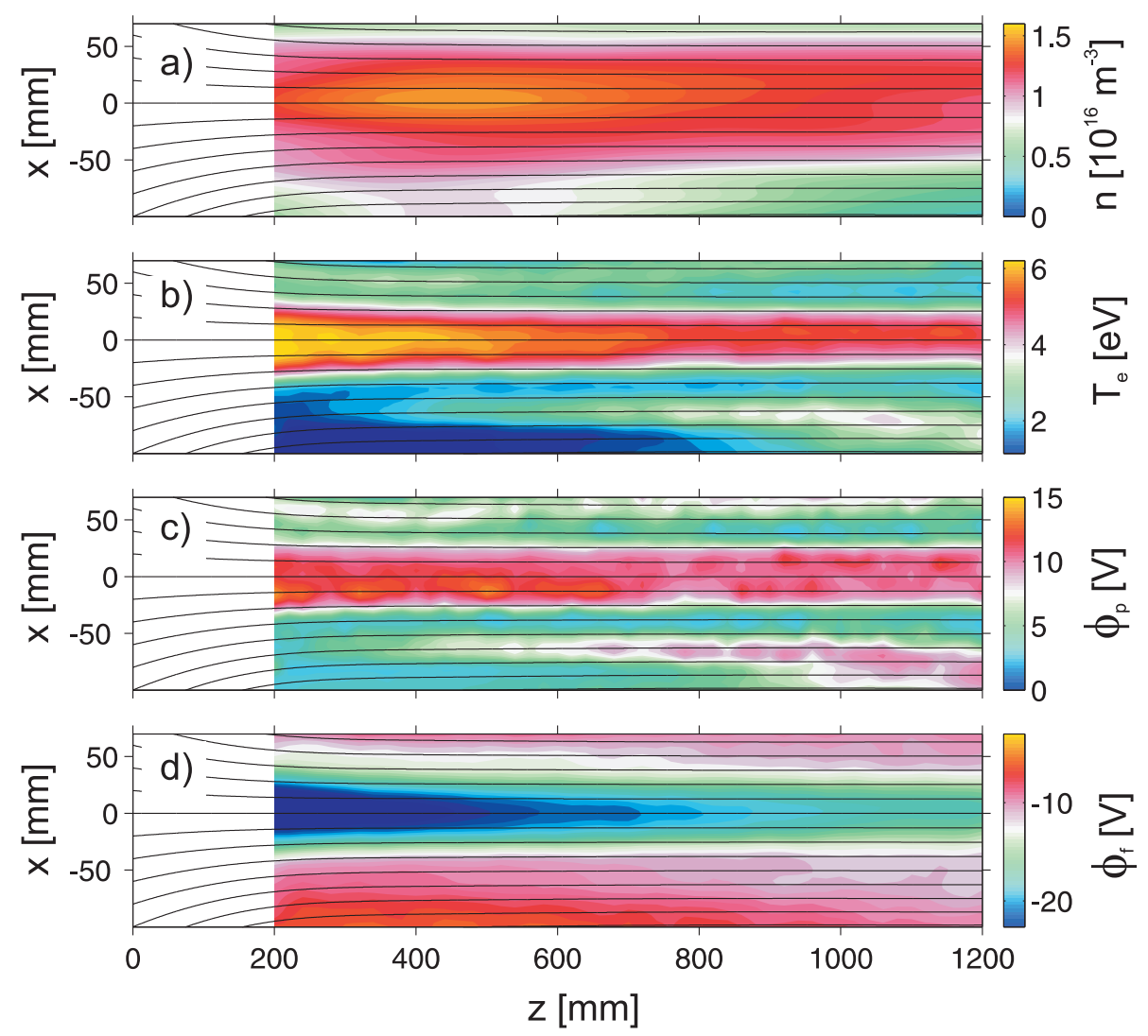

Figure 4. Radial axial profiles of plasma density (a), electron temperature (b) and plasma potential (c). The superimposed background magnetic field is superimposed as black contour lines. 
The resulting electric field is shown in Fig. 3(d) for an axial distance of $z=80 \mathrm{~mm}$ from the antenna. Due to the different shape of $B_{z}(r, z)$ in Fig. 3(a,b), the radial position of maximum power absorption is inwards shifted from $r=80 \mathrm{~mm}$ for the vacuum case to $r=50 \mathrm{~mm}$ in the plasma case.

\section{Spatial structure of plasma parameter profiles}

The profiles of plasma density, electron temperature, and plasma potential in the $(r, z)$ plane measured in the inductively coupled discharge with the spiral antenna are shown in Fig. 4. The operational parameters are listed in Tab. 1. For technical reasons, the measurements are taken for distances $z \geq 20 \mathrm{~cm}$ from the antenna. The spiral antenna is located at $z=0$ in a region of already fringing magnetic field lines, as indicated in Fig. 4 by the black lines. The electron density profile $n_{e}(r, z)$ in Fig. 4 (a) has a small peak at $z \approx 250 \mathrm{~mm}$ with $n_{e}=1.4 \cdot 10^{16} \mathrm{~m}^{-3}$. At larger distance from the antenna, the density is almost constant with $n_{e}=1 \cdot 10^{16} \mathrm{~m}^{-3}$. The electron temperature profile $T_{e}(r, z)$ shown in Fig. 4 (b) peaks close $z=200 \mathrm{~mm}$ at $T_{e} \approx 6 \mathrm{eV}$ and slightly decreases for larger distances to $T_{e} \approx 5 \mathrm{eV}$. The radial density profile is significantly broader than the electron temperature profile. At $x= \pm 50 \mathrm{~mm} T_{e}$ drops already to $2-3 \mathrm{eV}$. Note that only the high energetic electrons from the tail of the EEDF are subject to impact ionization. This causes a broadening of the density profile, if compared to the $T_{e}$-profile. Magnetic mapping is observed in the $T_{e}$-profile, which narrows along the field lines for increasing distance from the antenna. The plasma potential profile $\phi_{p}(r, z)$ in Fig. $4(\mathrm{c})$ is mainly determined by the sheath at the end plate and therefore exhibits more or less the same features as the electron temperature profile. The measured range $\phi_{p} \approx 10-15 \mathrm{~V}$ is in rough agreement with the relation $\phi_{p}=\phi_{f}+\alpha T_{e}$, where $\alpha=4.7$ for Argon and the central floating potential $\phi_{f}$ (not shown) varies gently between $-20 \mathrm{~V}$ at $z=200 \mathrm{~mm}$ and $-15 \mathrm{~V}$ for $z>800 \mathrm{~mm}$.

\section{Helicon-wave sustained discharges}

While plasma production with the spiral antenna at low $R F$ power is dominated by inductive coupling, a sudden increase of the plasma density is observed for higher $R F$ power. The plasma density, potential, and electron temperature for increasing $R F$-power $P_{R F}$ is shown in Fig. 5. The sudden jump to higher electron density and lower electron temperature at high $R F$-power is indicated with arrows. All major
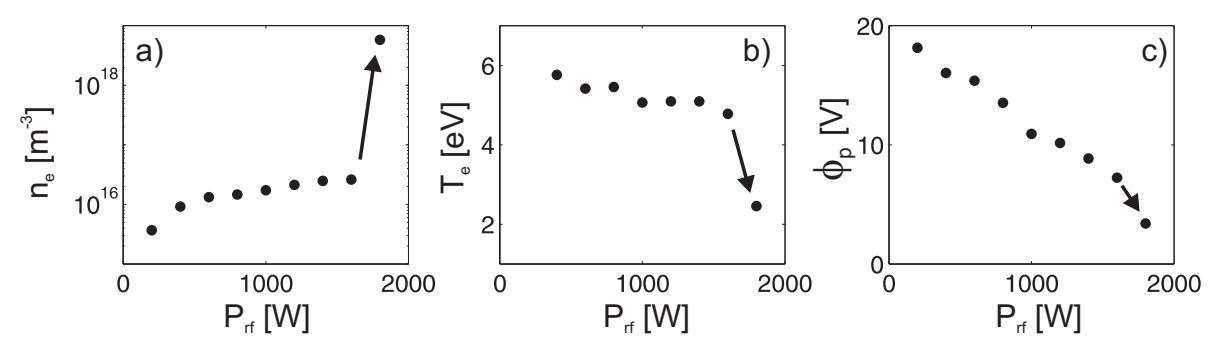

Figure 5. Evolution of central electron density $n_{e}(\mathrm{a})$, electron temperature $T_{e}$ (b) and plasma potential $\phi_{p}$ (c) for increasing $R F$ power $P_{r f}$. The other discharges parameters are constant $\left(p_{0}=0.4 \mathrm{~Pa}, B_{0}=60 \mathrm{mT}\right.$ 


\begin{tabular}{llll}
$B_{0}[\mathrm{mT}]$ & $n\left[10^{16} \mathrm{~m}^{-3}\right]$ & $k_{\|}[1 / \mathrm{m}]$ & $k_{\perp}[1 / \mathrm{m}]$ \\
\hline 40 & 3.7 & 0.89 & 29 \\
60 & 4.7 & 0.73 & 29 \\
80 & 5.5 & 0.69 & 23
\end{tabular}

Table 2. Density and wavenumbers for three different magnetic field strengths.

parameters are kept constant at $p_{0}=0.4 \mathrm{~Pa}, B_{0}=60 \mathrm{mT}$, and relative reflected power $P_{r f} / P_{\text {ref }} \approx 5 \%$ ). The measurements were made at the central axis $r=0$ at $z=500 \mathrm{~mm}$. In the inductive mode $\left(P_{R F}=200 \ldots 1600 \mathrm{~W}\right)$ the density increases from initially $n=4 \cdot 10^{15} \mathrm{~m}^{-3}$ to $n=2.6 \cdot 10^{16} \mathrm{~m}^{-3}$ about proportional with $P_{r f}$. Conversely the electron temperature decreases from $T_{e} \approx 6 \mathrm{eV}$ at $P_{R F}=200 \mathrm{~W}$ to $T_{e} \approx 4.5 \mathrm{eV}$ at $P_{R F}=1600 \mathrm{~W}$, which also causes a decrease of the plasma potential from $\phi_{p}=18 \mathrm{~V}$ to $\phi_{p}=8 \mathrm{~V}$. At a certain transition point, at $P_{R F} \approx 1.6 \mathrm{~kW}$, the plasma density suddenly increases by two orders of magnitude to $n \approx 3.9 \cdot 10^{18} \mathrm{~m}^{-3}$, while the electron temperature and plasma potential drop to $2.5 \mathrm{eV}$ and $3.5 \mathrm{~V}$, respectively. The density jump is accompanied by a bright blue emission core, due to the high population of ArII states. These are typical features of helicon wave sustained plasmas [10].

The parallel magnetic component $\tilde{B}_{z}$ of the helicon wave field has been measured using an interferometric setup. Measurements have been made in the inductive mode (antenna without Faraday shield) at three different magnetic field strengths. The spatio-temporal evolution of $\tilde{B}_{z}(z, t)$ and $\tilde{B}_{z}(r, t)$ for $B_{0}=80 \mathrm{mT}$ is shown in Fig. 6(a,b). The amplitude peaks on the central axis $r=0$, which is consistent with the structure of a helicon mode with azimuthal modenumber $m=0$, given for a uniform plasma profile by $\tilde{B}_{z} \sim k_{\perp} J_{0}\left(k_{\perp} r\right)$, with $k_{\perp}$ the transverse wavenumber and $J_{0}$ the zero order Bessel function of the first kind [11]. A polar plot of the measured normalized wavenumbers is shown in Fig. 6(c) for the three $B_{0}$ values.

The density and wavenumbers as estimated from the wavefield measurement are compiled in Table 2. The density is slightly higher if compared to the measurements with antenna with Faraday shield (cf. Fig. 5(a)). Note that the transverse wavenumber has been estimated from the radial profile of $\tilde{B}_{z}$. The values are close
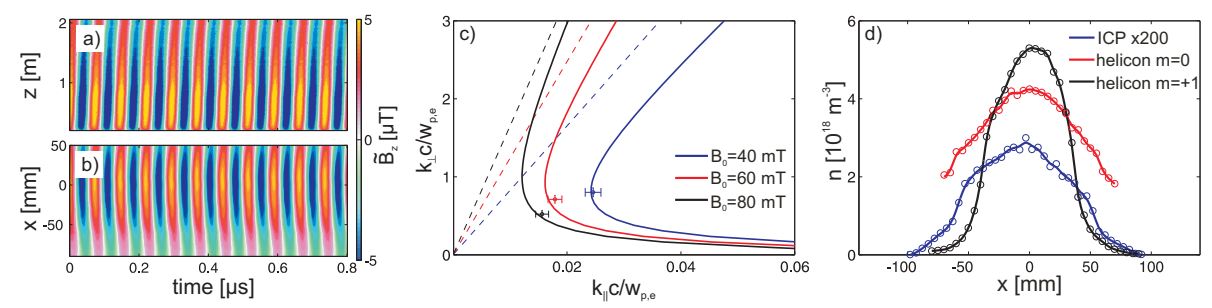

Figure 6. Spatio-temporal evolution of the magnetic component $\tilde{B}_{z}(z, t)$ (a) and $\tilde{B}_{z}(r, t)(\mathrm{b})$ of the helicon wave field using the spiral antenna. (c) Normalized polar plot of the $\mathrm{R}$-wave dispersion relation (solid) and the resonance cone given by $\cos \theta=\omega / \omega_{c, e}$ indicating the maximum angle of propagation (dashed) for different $B_{0}$ values. The measured wavenumbers (see text for details) are shown as dots. (d) Radial plasma density profile for the inductive (ICP) and helicon modes using the $m=0$ and $m=+1$ antenna. 
to the theoretically predicted value $k_{\perp}=3.83 / a \approx 30 \mathrm{~m}^{-1}$, where $a=12.5 \mathrm{~cm}$ is taken as maximum plasma radius in the inductive mode (cf. Fig. 6(b)). The measured wavenumbers can be compared to the R-wave dispersion in the cold plasma approximation [10],

$$
k^{2} c^{2}=\omega^{2}-\frac{\omega \omega_{p, e}^{2}}{\omega-\omega_{c, e} \cos \theta},
$$

where $\omega / 2 \pi=13.56 \mathrm{MHz}$ is the $R F$ frequency, $\omega_{p, e}$ the electron plasma frequency, $\omega_{c, e}$ the electron cyclotron frequency, $\cos \theta=k_{\|} / k$ and $k^{2}=k_{\perp}^{2}+k_{\|}^{2}$. A normalized polar plot of the dispersion relation calculated for the experimental parameters $n$ and $B_{0}$ is shown in Fig. 6(c). Within the error bars, a good agreement between the dispersion relation and the measured wavenumbers is found. Helicon waves can propagate in the operation regime in which the wavelength is large compared to the electron skin depth, $k c / w_{p, e} \ll 1$. In such a case, the dispersion yields a simple relation between the plasma density and the background magnetic field $k_{\|} k / \omega=n e \mu_{0} / B_{0}$. In the short wavelength regime, $k c / w_{p, e} \gg 1$, the quasi-electrostatic Trivelpiece-Gould modes are observed, but they are strongly damped [27]. The additional constraints for the helicon wave propagation on $n$ and $B_{0}$ can be expressed by two parameters, $\alpha=w_{p, e}^{2} /\left(w_{c, e}^{2} N^{2}\right)$ and $\beta=\omega \omega_{c, e} N^{2} /\left(\omega_{p, e}^{2}\right)$, which have to satisfy $\alpha<1 / 4$ and $\beta<1[28] . \stackrel{N}{N}=k_{\|} c / \omega$ is the refractive index. For our operational experimental parameters, the peak $\alpha$ and $\beta$ values are 0.24 and 0.05 , respectively. Hence, it can be concluded that the measured wavefield can be attributed to a helicon wave. A comparison of the radial plasma density profiles for the inductive, the $m=0$ helicon and the $m=+1$ helicon modes is shown in Fig. 6(d). The RFinput power in the helicon modes is $1.8 \mathrm{~kW}$, in the inductive mode $600 \mathrm{~W}$. For convenience the profile of the inductive mode of the spiral antenna is also shown $\left(P_{R F}=600\right)$. The peak density of the $m=+1$ helicon discharge is by a factor 1.4 larger if compared to the $m=0$ case. The FWHM width of the density profile increases from $80 \mathrm{~mm}(m=+1)$ to $140 \mathrm{~mm}(m=0)$.

\section{Summary}

A planar spiral induction coil has been installed in the VINETA device. The diameter of the spiral antenna $(20 \mathrm{~cm})$ is a factor of two larger than the previously used helical $m=+1$ antenna $(10 \mathrm{~cm})$. At low $R F$ power, the spiral antenna leads to inductive coupling with peak densities of $n \approx 1-5 \cdot 10^{16} \mathrm{~m}^{-3}$ and electron temperatures of $T_{e} \approx 5-6 \mathrm{eV}$. This reduces the Coulomb collisional by a factor of 100 in comparison to a helicon discharge. At higher $R F$ powers the coupling of the spiral antenna shows a sudden transition to a helicon sustained discharge mode. The transition is characterized by a density jump to $n \approx 3 \cdot 10^{18} \mathrm{~m}^{-3}$ and a drop of the electron temperature to $T_{e} \approx 2 \mathrm{eV}$, which is comparable to the standard $m=+1$ antenna operation. The wavefield of the helicon modes has been measured in the inductive discharge mode, where the wave's energy is not dissipated to the plasma particles and the influence of multiple reflections of the propagating wave fronts at the plasma boundaries can be neglected [29]. The radial profile of the $\tilde{B}_{z}$ component agrees well with the predicted structure of a $m=0$ helicon mode. The measured transverse wavenumber is in good agreement with the theoretical prediction, if the footpoint of the plasma density profile is taken as boundary condition. Comparing the wavefield measurements with the helicon dispersion relation, we find good agreement. Due to 
the larger excitation region in the $m=0$ helicon discharge the FWHM width of the plasma density profile increases from $80 \mathrm{~mm}(m=+1)$ to $140 \mathrm{~mm}(m=0)$.

\section{References}

[1] J. Hopwood. Review of inductively coupled plasmas for plasma procesing. Plasma Sources Sci. Technol., 1:109-116, 1992.

[2] JH Keller. Inductive plasmas for plasma processing. Plasma Sources Sci. Technol., 5(2):166172, MAY 1996.

[3] S Shinohara, S Takechi, N Kaneda, and Y Kawai. Helicon $\mathrm{m}=0$ mode characteristics in largediameter plasma produced by a planar spiral antenna. Plasma Phys. Controlled Fusion, 39(9):1479-1486, SEP 1997.

[4] C. M. Franck, O. Grulke, and T. Klinger. Transition from unbounded to bounded plasma whistler wave dispersion. Phys. Plasmas, 9(8):3254-3258, 2002.

[5] M Kramer, B Lorenz, and B Clarenbach. Helicon sources with $\mathrm{m}=1$ and $\mathrm{m}=2$ helical antenna coupling. Plasma Sources Sci. Technol., 11(3A, Sp. Iss. SI):A120-A130, AUG 2002. 25th International Conference on Phenomena in Ionized Gases, NAGOYA, JAPAN, JUL 17-22, 2001.

[6] E Speth, HD Falter, P Franzen, U Fantz, M Bandyopadhyay, S Christ, A Encheva, M Froschle, D Holtum, B Heinemann, W Kraus, A Lorenz, C Martens, P McNeely, S Obermayer, R Riedl, $\mathrm{R}$ Suss, A Tanga, R Wilhelm, and D Wunderlich. Overview of the RF source development programme at IPP Garching. Nucl. Fusion, 46(6):S220-S238, JUN 2006. 4th IAEA Technical Meeting on Negative Ion Based Neutral Beam Injectors, Padova, ITALY, MAY 09-SEP 11, 2005.

[7] C. Charles. Plasmas for spacecraft propulsion. J. Phys. D: Appl. Phys., 42(16), AUG 212009.

[8] A. R. Ellingboe and R. W. Boswell. Capacitive, inductive and helicon-wave modes of operation of a helicon plasma source. Phys. Plasmas, 3(7):2797-2804, 1996.

[9] R. W. Boswell. Plasma production using a standing helicon wave. Phys. Lett., 33A(7):457-458, 1970.

[10] R. W. Boswell. Very efficient plasma generation by whistler waves near the lower hybrid frequency. Plasma Phys. Controlled Fusion, 26(10):1147-1162, 1984.

[11] F. F. Chen. Plasma ionization by helicon waves. Plasma Phys. Controlled Fusion, 33(4):339364, 1991.

[12] JE Stevens, MJ Sowa, and JL Cecchi. HELICON PLASMA SOURCE EXCITED BY A FLAT SPIRAL COIL. J. Vac. Sci. Technol. A, 13(5):2476-2482, SEP-OCT 1995. 41st National Symposium of the American-Vacuum-Society, DENVER, CO, OCT 24-28, 1994.

[13] S Shinohara and T Tanikawa. Characteristics of a large volume, helicon plasma source. Phys. Plasmas, 12(4), APR 2005.

[14] C. M. Franck, O. Grulke, and T. Klinger. Mode transitions in helicon discharges. Phys. Plasmas, 10(1):323-325, 2003.

[15] F. F. Chen. Physics of helicon discharges. Phys. Plasmas, 3(5):1783-1793, 1996.

[16] J. P. Rayner, A. D. Cheetham, and G. N. French. Radio frequency matching for helicon plasma sources. J. Vac. Sci. Technol. A, 14(4):2048-2055, 1996.

[17] I. D. Sudit and F. F. Chen. RF compensated probes for high-density discharges. Plasma Sources Sci. Technol., 3:162-168, 1994

[18] V. I. Demidov, S. V. Ratynskaia, R. J. Armstrong, and K. Rypdal. Probe measurements of electron energy distributions in a strongly magnetized low-pressure helium plasma. Phys. Plasmas, 6:350-358, 1999.

[19] C. M. Franck, O. Grulke, and T. Klinger. Magnetic fluctuation probe design and capacitive pickup rejection. Rev. Sci. Instrum., 73(11):3768-3771, 2002.

[20] R. H. Lovberg. Plasma Diagnostic Techniques. Academic, New York, 1965.

[21] J. Hopwood, C. R. Guarnieri, S. J. Whitehair, and J. J. Cuomo. Electromagnetic fields in a radio-frequency induction plasma. J. Vac. Sci. Technol. A, 11(1):147-151, 1993.

[22] U Kortshagen, ND Gibson, and JE Lawler. On the E-H mode transition in RF inductive discharges. J. Phys. D: Appl. Phys., 29(5):1224-1236, MAY 141996.

[23] MM Turner and MA Lieberman. Hysteresis and the E-to-H transition in radiofrequency inductive discharges. Plasma Sources Sci. Technol., 8(2):313-324, MAY 1999.

[24] VA Godyak and RB Piejak. Abnormally low electron-energy and heating-mode transition in a low-pressure argon rf discharge at $13.56 \mathrm{mhz}$. Phys. Rev. Lett., 65(8):996-999, AUG 201990.

[25] LJ Mahoney, AE Wendt, E Barrios, CJ Richards, and JL Shohet. Electron-density and energy- 
distributions in a planar inductively-coupled discharge. J. Appl. Phys., 76(4):2041-2047, AUG 151994.

[26] C. Lechte, S. Niedner, and U. Stroth. Comparison of turbulence measurements and simulations in the low-temperature plasma in the torsatron TJ-K. New J. Phys, 4:34.1-34.16, 2002.

[27] KP Shamrai and VB Taranov. Volume and surface rf power absorption in a helicon plasma source. Plasma Sources Sci. Technol., 5(3):474-491, AUG 1996.

28] K. P. Shamrai, V. P. Pavlenko, and V. B. Taranov. Excitation, conversion and damping of waves in a helicon plasma source driven by an $\mathrm{m}=0$ antenna. Plasma Phys. Controlled Fusion, 39:505-529, 1997.

[29] C. M. Franck, O. Grulke, A. Stark, T. Klinger, E. E. Scime, and G. Bonhomme. Measurements of spatial structures of different discharge modes in a helicon source. Plasma Sources Sci. Technol., 2005. accepted for publication. 